\title{
Simultaneous Operation of a Raman Fiber Amplifier and Laser Pumped by a Dual-Wavelength $\mathrm{Nd}^{3+}$-Doped Fiber Laser
}

\author{
Yahua Li, Student Member, IEEE, Stuart D. Jackson, Yucheng Zhao, and Simon Fleming
}

\begin{abstract}
This paper presents an experimental study on the dynamics of Raman fiber lasers that use highly $\mathrm{GeO}_{2}$-doped fibers as an active medium and a dual-wavelength $(1060$ and $1090 \mathrm{~nm})$ $\mathrm{Nd}^{3+}$-doped fiber laser as a pump source. The 1090-nm pump wavelength is located within the $\mathrm{SiO}_{2}$ Raman gain spectrum relating to the $1060-\mathrm{nm}$ pump wavelength, and competition is observed between Raman amplification of the 1090-nm emission with the 1060-nm emission used as the pump source and Raman lasing, which is independent of the 1090-nm amplification and which is also uses the $1060-\mathrm{nm}$ emission as the pump source. Several pump configurations have been demonstrated to generate specific Stokes emissions generated through Raman lasing or amplification. Changing the gain-to-loss ratio by introducing intracavity loss of Raman emissions or increasing the Raman fiber length within each configuration can force either Raman amplification or lasing to dominate. The maximum slope efficiency as a function of the launched pump power was $\sim 55 \%$ with a total output power of $1.6 \mathrm{~W}$ produced. A red shift of both the pump and the Stokes wavelengths is experimentally observed when the launched diode pump power is scaled up.
\end{abstract}

Index Terms-Dual-pump, highly $\mathrm{GeO}_{2}$-doped, Raman fiber amplifier (RFA), Raman fiber laser (RFL), thermal effect.

\section{INTRODUCTION}

$\mathbf{S}$ TIMULATED Raman scattering (SRS), one of the wellknown nonlinear interactions that occurs in optical fibers, is used to generate a downshifted Stokes emission or amplify an injected signal. This phenomenon benefits from the long interaction length and the large light intensity associated with the small core dimension of the fibers. Recently, a variety of studies on Raman fiber lasers (RFLs) and Raman fiber amplifiers (RFAs) in single-mode fibers have been carried out [1]-[5]. Efficient operation of RFLs based on $\mathrm{P}_{2} \mathrm{O}_{5}$-doped silica binary fiber (PDF) has been demonstrated because of its large Stokes frequency shift compared with that of either $\mathrm{GeO}_{2}$-doped silica binary fiber (GDF) or pure silica fiber [6]-[9]. On the other hand, GDF is widely used in RFLs and RFAs for its higher Raman gain

Manuscript received November 4, 2004; revised February 3, 2005. The work of Y. Li was supported by the International Postgraduate Research Scholarship (IPRS), funded by the Commonwealth Department of Education, Science and Training (DEST), Australia. This work was supported by the Australian Research Council and the Australian Photonics Co-operative Research Centre.

Y. Li is with the Optical Fiber Technology Centre and the School of Electrical and Information Engineering, University of Sydney, Eveleigh NSW 1430, Sydney, Australia (e-mail: y.li@ oftc.usyd.edu.au).

S. D. Jackson, Y. Zhao, and S. Fleming are with the Optical Fiber Technology Centre, Australian Photonics CRC, University of Sydney, Eveleigh NSW 1430, Sydney, Australia.

Digital Object Identifier 10.1109/JLT.2005.846890 cross section, compared with PDF and silica fiber [6], [10], and is therefore useful for obtaining a higher Raman gain coefficient for a given level of pump power. Many wavelengths can also be generated when combining both the silica-related frequency shift and the phosphorus-related frequency shift [8] or in highsilica binary glasses with a combined PDF and GDF system [11]. For the first time, to the authors' knowledge, simultaneous operation of cascaded Raman fiber lasing and Raman fiber amplification is reported in highly $\mathrm{GeO}_{2}$-doped single-mode fibers when pumped by a dual-wavelength $\mathrm{Nd}^{3+}$-doped double-clad fiber lasers (DCFLs). The shorter 1060-nm pump wavelength acted as the pump source for its first Stokes emission and for the amplification of the longer 1090-nm pump wavelength, and hence competition between Raman amplification and lasing was experimentally observed. With careful control of the intensity of the individual pump wavelengths, the Stokes emissions relating to the dual pumps can be selected to the desired output wavelength. It is also demonstrated that the red shift in the central wavelength of the pump and Stokes emission related to the temperature increases in the core of the pump laser.

\section{EXPERIMENTAL SETUP}

In the experiment, we constructed three types of RFLs pumped by $\mathrm{Nd}^{3+}$-doped double-clad fiber lasers, the latter of which was forward-pumped by a high-power diode laser system at a wavelength of $805 \mathrm{~nm}$. The $\mathrm{Nd}^{3+}$-doped fiber was codoped with $\mathrm{Al}^{3+}$ and $\mathrm{Ge}^{4+}$, had an active core diameter of $4.4 \mu \mathrm{m}$ and a numerical aperture (NA) of 0.16 , which provided a 920-nm cutoff wavelength. The hexagonal-shaped pump core with a dimension of 300/260 $\mu \mathrm{m}$ for the longer/shorter axis was surrounded by a low-index ultraviolet (UV)-curable fluoro-polymer to create an NA of 0.35 for the pump light. The measured concentration and pump absorption coefficient of $\mathrm{Nd}^{3+}$ ions was $\sim 2200$ parts per million (ppm) and $0.04 \mathrm{~m}^{-1}$, respectively. A single-mode pigtail fiber (fiber P) was spliced with low loss to the output end of the DCFL to serve as a filter, removing most of the $805-\mathrm{nm}$ pump light. Fig. 1 schematically shows experimental configurations. The first configuration (called "Setup A") is shown in Fig. 1(a). A broadband dielectric mirror (M) butted against the input end to the $\mathrm{Nd}^{3+}$-doped fiber and Fresnel end reflection (FER) at the output end of fiber P (FER1) formed the resonator of the DCFL and emitted dual-wavelength output at $\sim 1060$ and $1090 \mathrm{~nm} \mathrm{[12].} \mathrm{The}$ output from the DCFL was collimated with a pair of lenses and launched into the single-mode GDF to serve as pump source for 


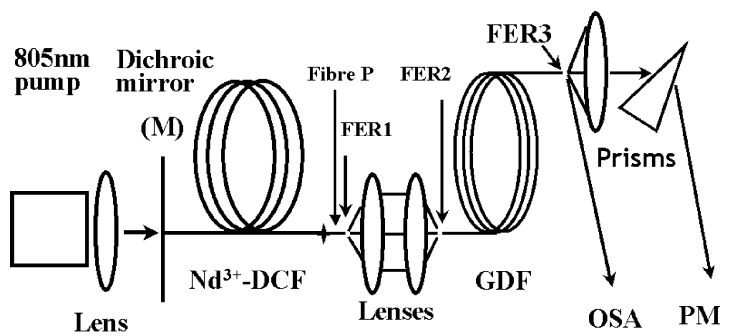

(a)

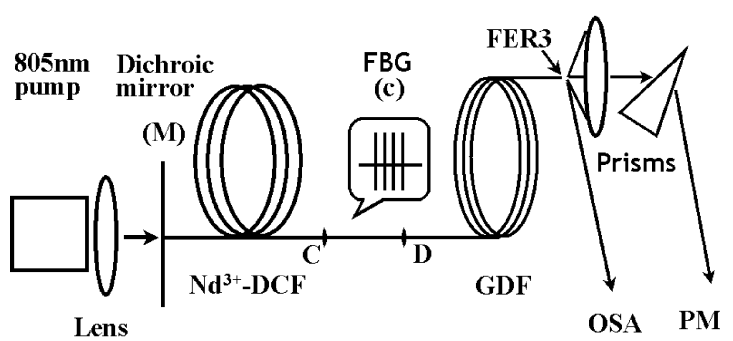

(b)

Fig. 1. Schematic diagram of the experimental setup of RFL (RFA) pumped by $\mathrm{Nd}^{3+}$-doped DCFL. (a) Setup A; (b) Setup B; and (c) Setup C, the same as Setup B with the exception that a 1060-nm FBG is inserted between C and D.

the RFLs and RFAs. In this setup, the cascaded RFLs used the resonator formed between M and FER3. As we show later, the cavity of the Raman fiber laser is separated to that of the DCFL. For the second configuration ["Setup B." see Fig. 1(b)], GDF fiber was directly spliced with low loss to the output end of fiber $\mathrm{P}$; therefore, both the pump lasers and the cascaded RFLs share the same resonator, which was formed between M and FER3. The reflectivity of the mirror $\mathrm{M}$ was estimated to be $100 \%$ from 1020 to $1190 \mathrm{~nm}$ and $18 \%$ from 1200 to $1300 \mathrm{~nm} .500 \mathrm{~m}$ of GDF (fiber 1) and $2 \mathrm{~km}$ of GDF (fiber 2) were made from the same preform with $18 \mathrm{~mol} \%$ concentration of $\mathrm{Ge}^{4+}$ ions. The fibers had a core diameter of $3.3 \mu \mathrm{m}$ with an NA of 0.25 , providing single-mode operation down to $1080 \mathrm{~nm}$. In the third configuration ["Setup C," see Fig. 1(b)], a fiber Bragg grating (FBG) with a reflectivity of $27 \%$ and bandwidth of $0.4 \mathrm{~nm}$ at the wavelength of $1060 \mathrm{~nm}$ was inserted between $\mathrm{C}$ and $\mathrm{D}$, as shown in Fig. 1(b). The pump resonator was therefore formed between $\mathrm{M}$ and the FBG and was separated from the cascaded RFLs. The output spectrum was measured with an Anritsu MS9710C optical spectrum analyzer (OSA). The individual power at each wavelength was measured with a Melles Griot 13PEM001 power meter (PM) after the output from the fiber laser was collimated and dispersed with the use of three prisms.

\section{RESULTS}

The output power at 1060 and $1090 \mathrm{~nm}$, labeled $P_{1060}$ and $P_{1090}$ (the subscript indicates the wavelength in nanometers), and the total $\sim 1-\mu \mathrm{m}$ output power, labeled $P_{T}$, exiting from the DCFL were measured after being dispersed with the use of three uncoated borosilicate prisms and are displayed in Fig. 2. Both $P_{1060}$ and $P_{1090}$ linearly increased with respect to the launched 805-nm pump. Fig. 3 shows the measured optical spectrum exiting from fiber 1 in Setup A at 3.2-W launched total pump power $P_{T}$. In addition to the residual pump light $P_{1060}$ and $P_{1090}$, there appeared emissions at 1112,1153 , and

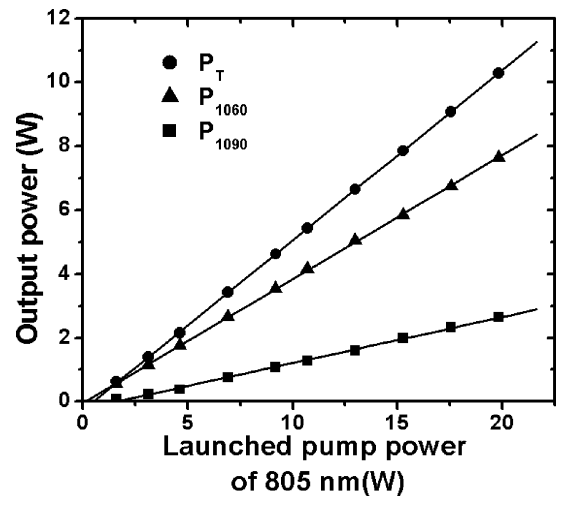

Fig. 2. Measured output power of $P_{1060}, P_{1090}$, and total at FER1 as a function of $805-\mathrm{nm}$ pump power launched into $\mathrm{Nd}^{3+}$ fiber.

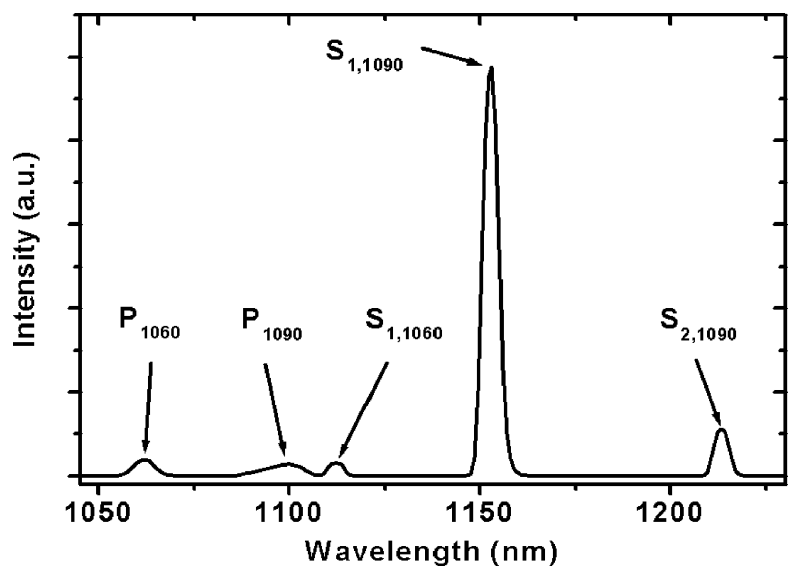

Fig. 3. Measured output spectrum exiting from fiber 1 in Setup A at 3.2-W launched pump power of $P_{T}$.

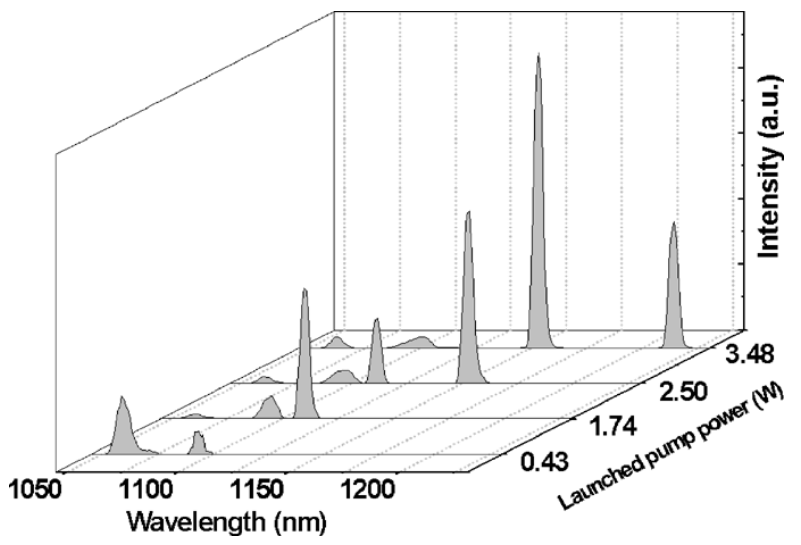

Fig. 4. Spectrum evolution exiting from fiber 1 in Setup A as a function of launched pump power of $P_{T}$.

$1214 \mathrm{~nm}$. The 1112-nm light $\left(S_{1,1060}\right)$ was determined to be the first Stokes shift relating to $P_{1060}$ through the Stokes shift of $\sim 13.3 \mathrm{THz}$. The 1153-nm $\left(S_{1,1090}\right)$ and 1214-nm $\left(S_{2,1090}\right)$ peaks were determined to be the first and second Stokes shifts corresponding to the pump light $P_{1090}$ generated through the same $\mathrm{GeO}_{2}$-related Stokes shift. The output spectrum evolution as a function of the launched pump power is shown in Fig. 4. Since the injected longer pump wavelength $\left(P_{1090}\right)$ is within the range of the $\mathrm{SiO}_{2}$-related Raman gain spectrum corresponding to the shorter pump wavelength $\left(P_{1060}\right), P_{1090}$ is initially 


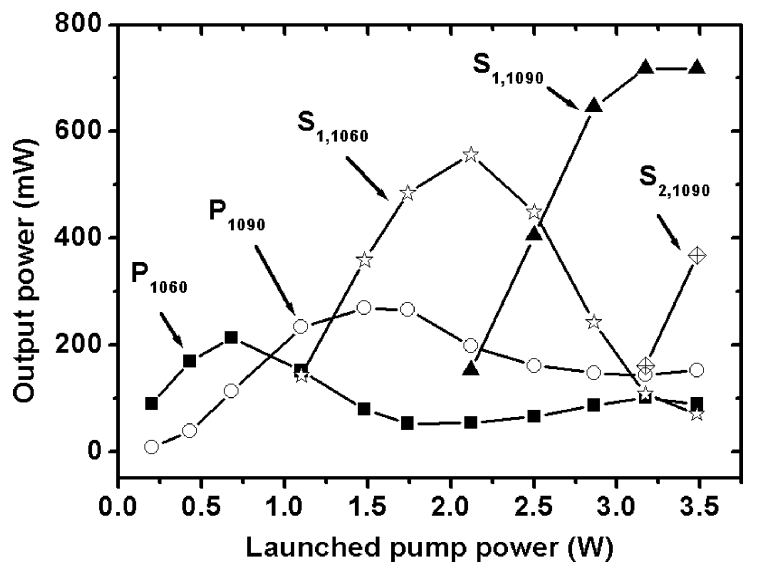

Fig. 5. Output power of $P_{1060}, P_{1090}, S_{1,1060}, S_{1,1090}, S_{2,1090}$ as a function of launched pump power of $P_{T}$ into fiber 1 in Setup A.

amplified due to SRS. Simultaneously, $P_{1060}$ also served as the pump light for the $S_{1,1060}$ emission relating to the $\mathrm{GeO}_{2}$ Raman gain. Because of the higher Raman gain of the $\mathrm{Ge}-\mathrm{O}$ vibration in the silica binary fiber, $S_{1,1060}$ can be generated easily. As a result, competition between Raman amplification of $P_{1090}$ and Raman Stokes lasing was observed at a 1.74-W launched pump power level (see Fig. 4). Increasing the launched pump power to $3.48 \mathrm{~W}$ provided $S_{1,1090}$ and $S_{2,1090}$ emissions, and $S_{1,1060}$ disappeared as shown in Fig. 4.

The output power at each wavelength exiting from fiber 1 was measured as a function of the launched pump power, as shown in Fig. 5. Initially, as the launched pump power is increased, the output of $P_{1060}$ decreases with respect to the $P_{1090}$ output as a result of Raman amplification. As mentioned previously, however, $P_{1060}$ is also acting as the pump source for its first $\mathrm{GeO}_{2}$-related Stokes shift, the $S_{1,1060}$ emission. As the launched power was increased, the $S_{1,1060}$ and $S_{1,1090}$ emissions reached their thresholds and oscillated. The power of these emissions increased dramatically while $P_{1060}$ further decreased. The experimental results are consistent with the theoretical simulation in [2] and the experiment described in [9]. The $S_{1,1090}$ starts to roll over as its power is depleted, pumping the $S_{2,1090}$ emission. The measured slope efficiencies for all three wavelengths, $S_{1,1060}, S_{1,1090}$, and $S_{2,1090}$, with respect to the launched pump power closely match at $\sim 67 \%$. This could be explained by the fact that the difference in quantum deficit at each pump and Stokes pair is small, and the gain and loss are near identical for these wavelengths.

Fig. 6 shows the spectrum evolution as a function of the launched pump power of fiber 2 for the same set of parameters as used for the generation of the results presented in Fig. 4. The Raman amplification process $\left(P_{1060}\right.$ pumping $\left.P_{1090}\right)$ was dominant and broadened and suppressed the $S_{1,1060}$ emission, which was observed in fiber 1 (see Fig. 4). This behavior can be explained by the fact that the factor-of-four increase in the length of fiber improves the Raman amplification, and Raman amplification of $P_{1090}$ is stronger than $S_{1,1060}$ generation, thereby allowing $P_{1090}$ to act as the pump source for further Stokes emissions $S_{1,1090}$ and $S_{2,1090}$. The behavior of these two lasing emissions is similar to those relating to $P_{1090}$ in fiber 1 .

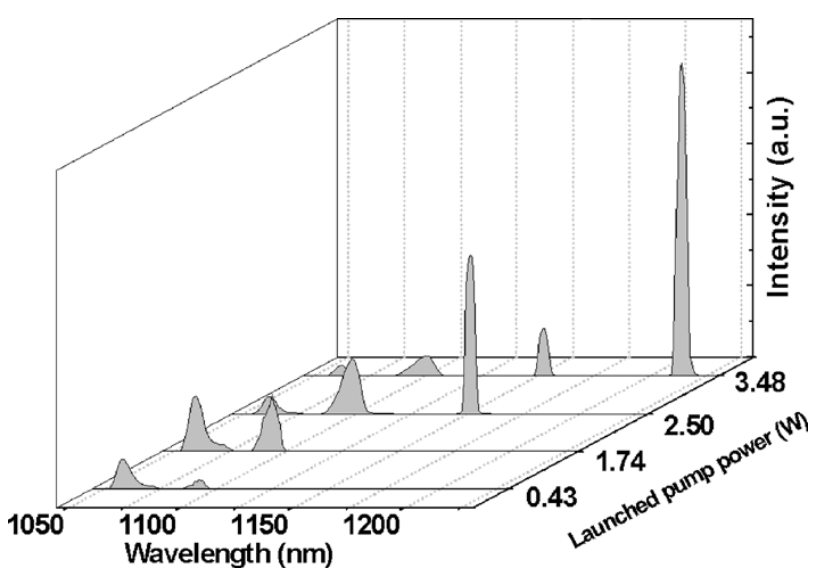

Fig. 6. Spectrum evolution exiting from fiber 2 in Setup A as a function of launched pump power of $P_{T}$.

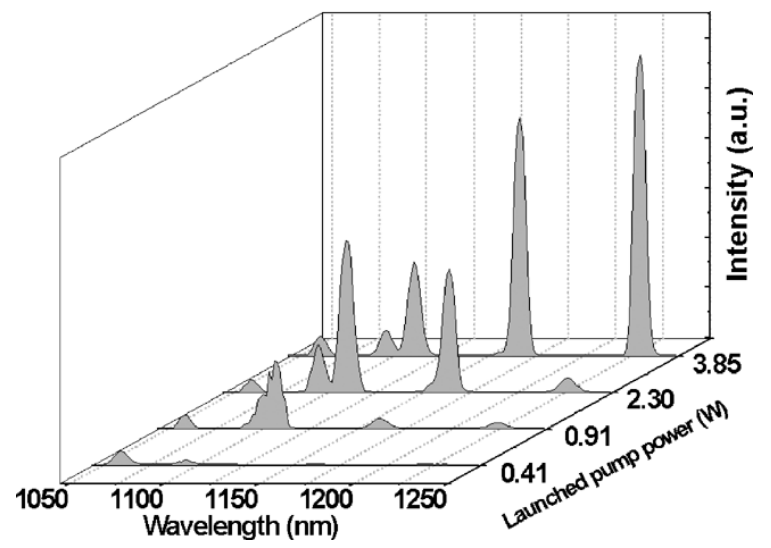

Fig. 7. Spectrum evolution exiting from fiber 1 in Setup B as a function of launched pump power of $P_{T}$.

The output spectrum evolution versus the launched pump power from fiber 1 in Setup B is shown in Fig. 7. The spectrum development is different to that which occurred in Setup A. In this configuration, both of the pump lines and the Stokes emissions oscillate together within the same resonator, and as a result the individual emissions can strongly affect each other. Since the total loss in Setup B is $\sim 4.6 \mathrm{~dB}$ lower than that in Setup A for fiber 1, amplification of $P_{1090}$ was suppressed after $S_{1,1060}$ appeared. For further increase in the launched pump power, the 1164-nm $\left(S_{2,1060}\right)$ and the 1230-nm $\left(S_{3,1060}\right)$ emission, corresponding to $P_{1060}$, appeared. The $S_{3,1060}$ emission is the highest order Stokes shift observed in the experiment. We also investigated the bandwidth of the individual Stokes emissions as functions of the launched pump power in the three different configurations. Fig. 8 shows the experimental results measured from fiber 1 in Setup B. It is noted that the bandwidth of the Stokes emissions is proportional to the launched pump power and that these bandwidths are narrower than the dual-pump wavelengths. This may be attributed to the narrow-gain bandwidth of the $\mathrm{GeO}_{2}$-related Raman spectrum.

The total output powers from fiber 1 and fiber 2 in Setup A and fiber 1 in Setup B as functions of the launched pump power level are shown in Fig. 9. The total power exiting from fiber 1 in Setup B is the highest of the three cases with a slope efficiency of $\sim 55 \%$, and fiber 2 in Setup A provided the lowest output 


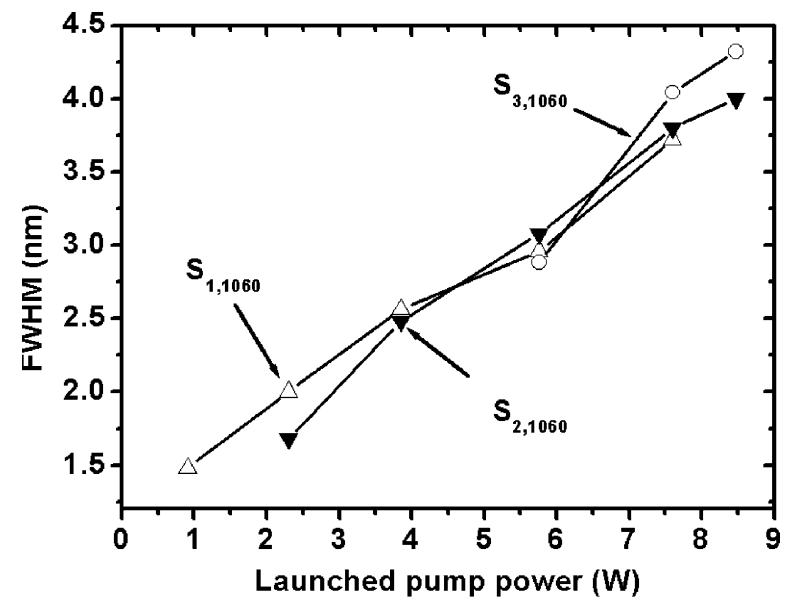

Fig. 8. Measured bandwidths of the individual Stokes emissions exiting from fiber 1 versus launched pump power of $P_{T}$ in Setup B.

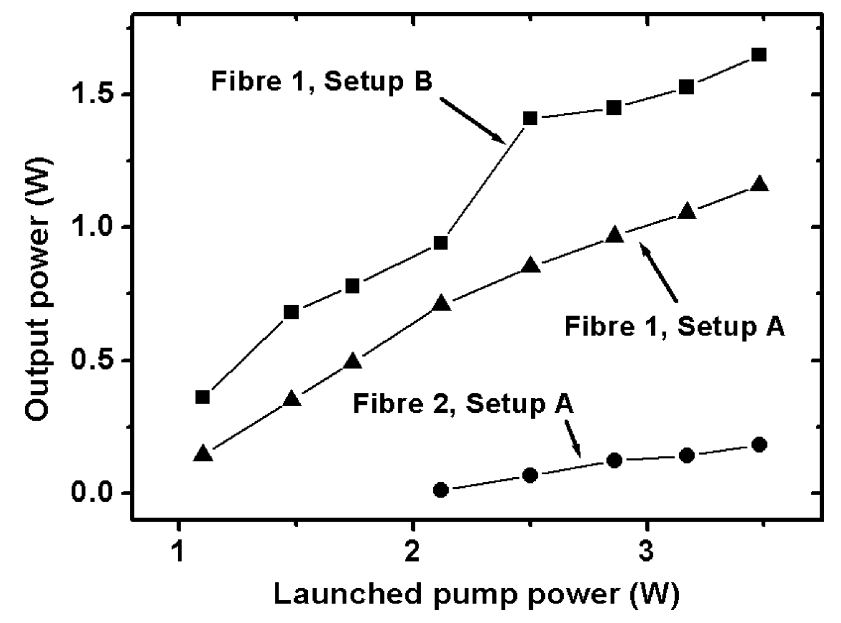

Fig. 9. Measured total output power for fiber 1 and fiber 2 in Setup A and fiber 1 in Setup B as a function of the launched pump power of $P_{T}$.

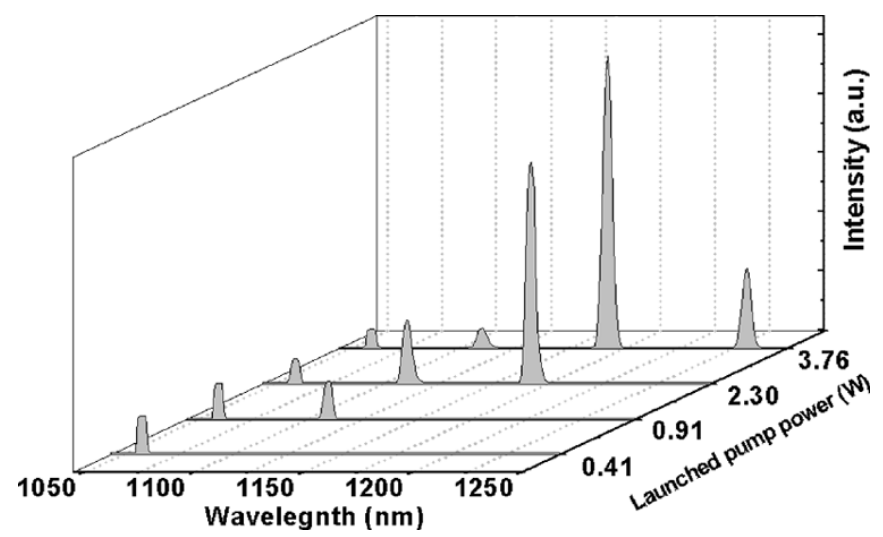

Fig. 10. Spectrum evolution exiting from fiber 1 in Setup $\mathrm{C}$ as a function of the launched pump power of $P_{T}$ ( $P_{1060}$ only).

power and a slope efficiency of $\sim 12 \%$. With regard to fiber 1 , this can be attributed to the lower intracavity loss in Setup B compared with that in Setup A. Due to the longer fiber length of fiber 2, however, the increase in the loss will outweigh that of the gain and thus lower the output power.

Fig. 10 shows the spectrum evolution measured in Setup C. Since a $0.4-\mathrm{nm}$ bandwidth FBG with a central wavelength of
$1060 \mathrm{~nm}$ was incorporated in the resonator, the $\mathrm{Nd}^{3+}$-doped DCFL was therefore forced to oscillate at the wavelength of $1060 \mathrm{~nm}$ only. The third order of Stokes emission was observed when the launched pump power $\left(P_{1060}\right.$ only) reached $3.76 \mathrm{~W}$.

\section{Discussions}

We have shown that optical amplification and lasing due to SRS can run simultaneously in a highly $\mathrm{Ge}^{4+}$-doped singlemode fiber using a dual-frequency pump source. In order to efficiently generate cascaded RFLs, the fiber used in the experiments was made with a small effective core area of $12 \mu \mathrm{m}^{2}$, a high Raman gain coefficient $(\sim 10.1 \mathrm{~dB} / \mathrm{km} \mathrm{W})$ and a low loss of 3-4 dB/km in the range of the working wavelengths. As a result, the cascaded RFL can be easily generated without incorporating pairs of FBGs. In a previous work [12], the Raman fiber used in the experiment had a large effective core area of 38 $\mu \mathrm{m}^{2}$, and the Raman gain coefficient was nine times less than that used in the current experiment. As a result, only Raman amplification was observed, and no Stokes emission was obtained in the $\mathrm{Nd}^{3+}$-doped silica fiber.

We established from the experiment that the RFL resonator relevant to Setup A is the same as that in Setup B, i.e., it is formed between $M$ and FER3. In the Setup A, the intracavity loss caused by the pair of lenses and FER is $4.6 \mathrm{~dB}$, and the effective reflectivity as a result of the round trip through the coupling lenses and $\mathrm{Nd}^{3+}$-doped fiber is calculated to be $12 \%$, higher than FER2. With the use of this simple configuration, one can control the intracavity loss and therefore examine the dependence of the output wavelength on the gain-to-loss ratio. In a free-running system using a broadband output coupler, with the use of this configuration, we can control the output wavelength as follows:

1) by varying the relative intensities of the two pumps;

2) by controlling the level of loss between the pump sources and cascaded RFLs;

3) by changing the length of the fiber.

Clearly, more theoretical work is required to fully understand this dual-pump RFL system, and a model for this system is currently under investigation.

Slight red shifts in the central wavelength of both the pump and the Stokes emissions were observed in all the configurations, when the launched diode pump power was increased. The red shift is related to a phase change in the lasing mode through two thermal effects [13]-[16]. The temperature increase inside the cavity when the launched pump power is scaled up will lead not only to a thermal expansion of the fiber, but also to a negative change in the refractive index. Both changes affect the stationary resonance condition. The change in the optical path length due to thermal expansion is, however, ten times greater than that due to the refractive-index change and hence dominates the thermally induced red shift [15]. In our case, the lasing wavelengths relate to the stationary resonance condition

$$
m_{i} \lambda_{i}=2 n_{i} d\left(1+\frac{1}{n_{i}} \cdot \frac{\partial n_{i}}{\partial T} \cdot \Delta T\right) \cdot\left(1+\frac{1}{d} \cdot \frac{\partial d}{\partial T} \cdot \Delta T\right)_{(1)}
$$

where $m$ is an integer relating to each longitudinal lasing mode, $i$ is an integer relating to the lasing wavelength, $n_{i}$ and $d$ are the 
TABLE I

VALUES OF SELECTED OPTICAL AND THERMAL PARAMETERS OF THE DCFL FIBER USED

\begin{tabular}{ll} 
Maximum $P_{L D}(\mathrm{~W})$ & 50 \\
$\eta_{\text {lamci }}$ & 0.7 \\
Core radius, $r_{0}(\mu \mathrm{m})$ & 2.2 \\
Fibre radius, $r_{I}(\mu \mathrm{m})$ & 150 \\
Fibre length, $d(\mathrm{~m})$ & 43 \\
Refiactive index, $n$ & 1.52 \\
$\partial n / \partial T$ & $-10[15]$ \\
$\left(\times 10^{-\tau} /{ }^{\circ} \mathrm{C}\right)$ & \\
Thermal expansion $\left(\times 10^{-} /{ }^{\circ} \mathrm{C}\right)$ & $87[15]$ \\
$4-20^{\circ} \mathrm{C}$ & \\
Thermal conductivity, $k\left(\mathrm{~W} / \mathrm{m} /{ }^{\circ} \mathrm{C}\right)$ & $1.38[16]$ \\
Heat transfer coefficient, $\alpha H\left(\mathrm{~W} / \mathrm{m}^{2} /{ }^{\circ} \mathrm{C}\right)$ & $52[17]$ \\
Radiative efficiency, $\eta_{\text {radiativs }}$ & $0.55[17]$ \\
\hline
\end{tabular}

refractive index of the individual lasing wavelengths and length of the cavity, respectively. If we assume that all longitudinal modes experience the same refractive index, then we obtain the overall red shift of all lasing modes from the calculation of the red shift relevant to each individual longitudinal lasing mode. For a single longitudinal mode, the red shift $\Delta \lambda_{\text {shift }}$ caused by the pump-induced temperature change $\Delta T$ is governed by [15]

$$
\Delta \lambda_{\text {shift }}=\lambda \cdot\left(\frac{1}{n} \cdot \frac{\partial n}{\partial T}+\frac{1}{d} \cdot \frac{\partial d}{\partial T}\right) \cdot \Delta T
$$

and $\Delta T$ can be approximated by [17]

$$
\begin{aligned}
\Delta T & \approx \frac{P_{\text {thermal }}}{2 \pi d}\left[\frac{1}{k} \ln \left(\frac{r_{1}}{r_{0}}\right)+\frac{1}{r_{1} \alpha_{H}}\right] \\
P_{\text {thermal }} & =P_{\mathrm{LD}} \cdot \eta_{\text {launch }}\left(1-\eta_{\text {radiative }}\right)
\end{aligned}
$$

where $k$ and $\alpha_{H}$ are the thermal conductivity and the heat transfer coefficient of the fiber, $r_{0}$ and $r_{1}$ are the radius of the fiber core and cladding, $d$ is the fiber length, $P_{\mathrm{LD}}$ is the power of the diode laser, $\eta_{\text {launch }}$ is the pump coupling efficiency, and $\eta_{\text {radiative }}$ is radiative emission efficiency of the pump laser. Since the pump-induced heat is distributed along the entire $\mathrm{Nd}^{3+}$-doped fiber, we consider the average temperature difference in (3). Based on the parameters provided in Table I, the increase to the maximum diode pump power of $50 \mathrm{~W}$ leads to an average temperature rise of $7.4 \mathrm{~K}$, resulting in a calculated red shift of $0.06 \mathrm{~nm} / \mathrm{K}$. The measured red shift as a function of the launched pump power (temperature) was $0.032 \mathrm{~nm} / \mathrm{W}$ $(0.05 \mathrm{~nm} / \mathrm{K})$. The experimental result is consistent with the simulation, and these figures are of the same order of magnitude as those obtained for phosphate glass [15].

\section{CONCLUSION}

In conclusion, a Raman fiber amplifier (RFA) and Raman fiber laser (RFL) device was successfully constructed using a highly $\mathrm{Ge}^{4+}$-doped fiber medium and dual-wavelength pumping. The 1060-nm wavelength acted as the pump source for both Stokes emission and the amplification of the longer 1090-nm wavelength. The measured overall slope efficiency from fiber 1 in Setup B with respect to the launched pump power was $\sim 55 \%$ with a maximum total output power of $1.6 \mathrm{~W}$.
A red shift of both of the pump and the Stokes emissions was experimentally observed, and the Stokes emission linewidth was proportional to the launched pump power when no gratings are used. With careful control, the gain-to-loss ratio of individual pumps by introducing the intracavity loss or increasing the fiber length, we can force either Raman amplification or lasing to select the desired output wavelength.

\section{ACKNOWLEDGMENT}

The authors would like to thank the Australian Photonics Cooperative Research Centre for financial support and T. Ryan of the Optical Fiber Technology Centre, University of Sydney, Sydney, Australia, for his fiber fabrication skills.

\section{REFERENCES}

[1] E. M. Dianov and A. M. Prokhorov, "Medium-power CW Raman fiber laser," IEEE, J. Sel. Topics Quantum Electron., vol. 6, no. 6, pp. 1022-1028, Nov.-Dec. 2000.

[2] S. D. Jackson and P. H. Muir, "Theory and numerical simulation of $n$ th-order cascaded Raman fiber lasers," J. Opt. Soc. Amer. B, Opt. Phys., vol. 18, pp. 1297-1306, 2001.

[3] M. Rini, I. Cristiani, and V. Degiorgio, "Numerical modeling and optimization of cascaded CW Raman fiber lasers," IEEE J. Quantum Electron., vol. 36, no. 10, pp. 1117-1122, Oct. 2000.

[4] M. Krause, S. Cierullies, H. Renner, and E. Brinkmeyer, "Design of widely tunable Raman fiber lasers supported by switchable FBG resonators," Electron. Lett., vol. 39, pp. 1795-1797, 2003.

[5] Z. Xiong, N. Moore, Z. G. Li, and G. C. Lin, "10-W Raman fiber lasers at $1248 \mathrm{~nm}$ using phosphosilicate fibers," J. Lightw. Technol., vol. 21, no. 10 , pp. 2377-2381, Oct. 2003.

[6] N. Shibata, M. Horigudhi, and T. Edahiro, "Raman spectrum of binary high-silica glasses and fibers containing $\mathrm{GeO}_{2}, \mathrm{P}_{2} \mathrm{O}_{5}$ and $\mathrm{B}_{2} \mathrm{O}_{3}$," $J$. Non-Cryst. Solids, vol. 45, pp. 115-126, 1981.

[7] M. Prabhu, N. S. Kim, L. Jianren, and K. Ueda, "Simultaneous two-color CW Raman fiber laser with maximum output power of $1.05 \mathrm{~W} / 1239 \mathrm{~nm}$ and $0.95 \mathrm{~W} / 1848 \mathrm{~nm}$ using phosphosilicate fiber," Opt. Commun., vol. 182, pp. 305-309, 2000.

[8] E. M. Dianov, I. A. Bufetov, M. M. Bubnov, M. V. Grekov, S. A. Vasiliev, and O. I. Medvedkov, "Three-cascaded 1407-nm Raman laser based on phosphorus-doped silica fiber," Opt. Lett., vol. 25, pp. 402-404, 2000.

[9] K. Suzuki, K. Noguchi, and N. Uesugi, "Selective stimulated Ramanscattering in highly $\mathrm{P}_{2} \mathrm{O}_{5}$-doped silica single-mode fibers," Opt. Lett., vol. 11, pp. 656-658, 1986.

[10] F. L. Galeener, J. C. Mikkelsen Jr., R. H. Geils, and W. J. Mosby, "The relative Raman cross sections of vitreous $\mathrm{SiO}_{2}, \mathrm{GeO}_{2}, \mathrm{~B}_{2} \mathrm{O}_{3}$, and $\mathrm{P}_{2} \mathrm{O}_{5}$," Appl. Phys. Lett., vol. 32, pp. 34-36, 1978.

[11] J. Xu, M. Prabhu, C. Li, J. Lu, and K. Ueda, "Output characteristics of a fiber Raman laser with a composite $\mathrm{GeO}_{2}$ and $\mathrm{P}_{2} \mathrm{O}_{5}$-doped silica fiber,' J. Modern Opt., vol. 48, pp. 1269-1279, 2001.

[12] S. D. Jackson and Y. H. Li, "Multiwavelength diode-cladding-pumped $\mathrm{Nd}^{3+}$-doped germano-aluminosilicate fiber laser," IEEE J. Quantum Electron., vol. 39, no. 9, pp. 1118-1122, Sep. 2003.

[13] J. Nilsson, S. Alam, J. A. Alvarez-Chavez, P. W. Turner, W. A. Clarkson, and A. B. Grudinin, "High-power and tunable operation of ErbiumYtterbium co-doped cladding-pumped fiber lasers," IEEE J. Quantum Electron., vol. 39, no. 8, pp. 987-994, Aug. 2003.

[14] G. Perna, V. Capozzi, M. Ambrico, and D. Smaldone, "Temperature dependence of the red shift and broadening of the exciton line in CdSe/GaAs laser ablated heterostructures," J. Lumin., vol. 76-77, pp. 534-539, 1998.

[15] Z. P. Cai, A. Chardon, H. Y. Xu, P. Feron, and G. M. Stephan, "Laser characteristics at $1535 \mathrm{~nm}$ and thermal effects of an Er:Yb phosphate glass microchip pumped by Ti:Sapphire laser," Opt. Commun., vol. 203, pp. 301-313, 2002.

[16] M. K. Davis, M. J. F. Digonnet, and R. H. Pantell, "Thermal effects in doped fibers," J. Lightw. Technol., vol. 16, no. 6, pp. 1013-1023, Jun. 1998.

[17] W. S. Man, Y. Z. Xu, H. Y. Tam, and A. S. Demokan, "Frequency instability in $\mathrm{Er} / \mathrm{Yb}$ fiber grating lasers due to heating by nonradiative transitions," IEEE Photon. Technol. Lett., vol. 11, no. 11, pp. 1390-1392, Nov. 1999. 


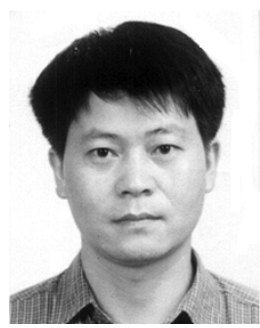

Yahua Li (S'03) received the B.E. degree and the M.M. degree from Huazhong University of Science and Technology (HUST), Wuhan, China, in 1988 and 2001, respectively. Currently, he is studying towards the Ph.D. degree in rare-earth-doped fiber lasers and Raman fiber lasers, at the Optical Fiber Technology Centre and the School of Electrical and Information Engineering, the University of Sydney, Sydney, Australia.

From 1988 to 2002, he worked at the National Research Center for Laser Technology and Engineering, HUST, where, in 1998, he became an Associate Professor. He was also a Section Manager, responsible for R\&D and marketing, at Huagong Laser Engineering Company, Ltd., Wuhan, China. His research interests include high-power solidstate lasers for biomedical and commercial applications.

Stuart D. Jackson received the B.Sc. degree and the B.Sc. (Hons.) degree from the University of Newcastle, Australia, in 1989 and 1990, respectively. He received the Ph.D. degree from the Centre for Lasers and Applications at Macquarie University, Sydney, Australia, in 1996.

In 1995, he joined the Laser Photonics Group at the University of Manchester. Manchester, U.K., where he initiated research into high-power fiber lasers. In 1999, he joined the Optical Fiber Technology Centre, University of Sydney, Sydney, Australia, where he is now an Australian Research Fellow sponsored directly by the Australian Research Council. His interests are in diode-pumped solid-state lasers, spectroscopy, and philosophy.
Yucheng Zhao received the B.S. degree in physics from Hebei Normal University, China, in 1990. He received the M.E. degree in electrical engineering from Harbin Engineering University, China, in 1993. He received the Ph.D. degree in optical communications from Northern Jiaotong University, China, in 1997.

In 1997, he joined the Institute of Lightwave Technology at Northern Jiaotong University, working on OTDM systems and fiber lasers. In 2000, he joined the Optoelectronics Research Centre at University of Southampton, Southampton, Australia, as a Postdoctoral Research Fellow working on microsilica fiber resonator. Since 2001, he has been with the Optical Fiber Technology Centre at the University of Sydney, Sydney, Australia, where he has been engaged in research on Raman fiber lasers. His interests also include fiber characterization and nonlinear signal processing.

Simon Fleming, photograph and biography not available at the time of publication. 